\title{
Relativistic and nonrelativistic bound states of the isotonic oscillator by Nikiforov-Uvarov method
}

\author{
Sameer M. Ikhdair ${ }^{1, *}$ and Ramazan Sever ${ }^{2}$, \\ ${ }^{1}$ Department of Physics, Near East University, Nicosia, North Cyprus, Turkey \\ ${ }^{2}$ Department of Physics, Middle East Technical University, 06800, Ankara,Turkey
}

(Dated: July 13, 2021)

\begin{abstract}
A nonpolynomial one-dimensional quantum potential in the form of an isotonic oscillator (harmonic oscillator with a centripetal barrier) is studied. We provide the non-relativistic bound state energy spectrum $E_{n}$ and the wave functions $\psi_{n}(x)$ in terms of the associated Laguerre polynomials in the framework of the Nikiforov-Uvarov method. Under the spin and pseudospin symmetric limits, the analytic eigenvalues and the corresponding two-component upper- and lower-spinors of the Dirac particle are obtained, in closed form.
\end{abstract}

Keywords: Schrödinger equation, Dirac equation, spin and pseudospin symmetry, harmonic oscillator, isotonic oscillator, self-adjoint operator, Nikiforov-Uvarov method.

PACS numbers: 03.65.Pm; 03.65.Ge; 02.30.Gp

${ }^{*}$ E-mail: sikhdair@neu.edu.tr

${ }^{\dagger}$ E-mail: sever@metu.edu.tr 


\section{INTRODUCTION}

The exact solutions of the nonrelativistic and relativistic wave equations are only possible in a few simple potential cases such as the Coulomb, the harmonic oscillator, the pseudoharmonic, isotonic potentials and others [1-4]. The most interesting and best known system inside this small family is the harmonic oscillator whose energy spectrum consists of an infinite set of equidistant energy levels. Many other oscillators, as for example harmonic oscillators perturbed by a term containing a fourth or a sixth power in the coordinate, have been extensively studied. Nevertheless, it is known the existence of another solvable onedimensional model which shares several interesting properties with the harmonic oscillator, the so-called isotonic oscillator whose spectrum coincides with that of the harmonic oscillator [5]. This potential model is considered as one of the most used models for the study of the dynamics of nonlinear systems. In particular, the energy spectrum of the singular potential is considerd as an isomorphous to the harmonic oscillator spectrum. Very recently, Fellows and Smith [6] have used the ideas of the factorization and supersymmetric (SUSY) approach $[7,8]$ to study the singular superpotentials and they proved that most of these oscillatory potentials are other partner potentials of the harmonic oscillators and derived an infinite set of exactly soluble potentials. A discussion of the supersymmetric connection between harmonic and isotonic oscillators can be traced in Ref. [9].

The isotonic potential takes the form,

$$
U_{\text {Isot }}(x)=U_{0}(x)+U_{g}(x)=\frac{1}{2} M \omega^{2} x^{2}+\frac{1}{2} \frac{g}{x^{2}}, x \neq 0, g \geq 0,
$$

where $\omega$ is the angular frequency of oscillator, $M \omega^{2}=K$ in classical mechanics and $g=$ $m(m+1)$. The common feature of this potential is that it consists of a harmonic term plus an additional rational function (centripetal barrier) which falls off at infinity like a constant $g$ times $1 / x^{2}$ with one regular singularity at $x=0$ along the whole domain $-\infty<x<\infty$.[1] It is clear from $(1)$ that $U_{g}(x)$ exhibits strong singularity when $x=0$, so that the wave functions must vanish at such a point. It is worthy to note that the Hilbert space associated to the models with $U_{g}(x)$ is narrowed as compared to the Hilbert space of the hamiltonian with potential $U_{0}(x)$. In addition, the domain of the hamiltonian associated to harmonic

[1] Centrifugal barrier does not make physical sense in one-dimension. It is often used for such singular terms in a potential. 
oscillator extends along the whole real axis $(-\infty, \infty)$, however, for potential (1) reduces itself to the half-line $(x \geq 0$ or $x \leq 0)$. The harmonic oscillator $U_{0}(x)$ and the isotonic oscillator $U_{\text {Isot }}(x)$ are plotted in Figure 1 for the sake of comparison. The aim is to show that both curves coincide for wide range of $x>0$ except for values of $x$ in the neighborhood of the vertical asymptotic line $x=0$ where the isotonic oscillator goes to infinity. They have also identical spectrum, however, shifted by two units. We consider this potential in the interval $(0, \infty)$ as in the case of (1). Authors of Refs. [7-9] have solved the Schrödinger equation for the potential (1) with $\omega=M=1$ and $g=2(m=1)$ using SUSY approach $[7,8]$ on the assumption that the superpotential $W(x)$ obtained from the wave function of the harmonic oscillator $\phi_{1}(x)$ can generate twice the potential (1) (see e.g., [6]). The singular term in the above potential is often called the centripetal barrier potential. However, the centripetal barrier potential here makes no physical sense in one dimension since the term $m(m+1) / x^{2}$ singularities are often related to the radial equation for the three-dimensional harmonic oscillator.

In this article we set up to present a study of the exact analytic nonrelativistic bound state energy spectrum and the correspoding wave functions in terms of the associated Laguerre polynomials $L_{n}^{\alpha}(z)$ (or the Kummer confluent hypergeometric function, $M(a, b, z)=$ $\left.{ }_{1} F_{1}(a ; b ; z)\right)$ by applying the Nikiforov-Uvarov (NU) method [10]. Overmore, we extend our study to investigate this potential model in the context of the spin and pseudospin symmetric Dirac equation [11-17]. In the presence of the spin symmetry $S \sim V=U_{\text {Isot }}(x)$ and pseudospin symmetry $S \sim-V=U_{\text {Isot }}(x)$, we investigate the exact $s$-wave bound state energy eigenvalues and corresponding upper and lower spinor wave functions in a systematic form [12-15]. We also show that the spin (pseudospin) symmetric Dirac solutions can be reduced to the $S=V=U_{\text {Isot }}(x)\left(S=-V=U_{\text {Isot }}(x)\right)$ in the presence of exact spin symmetry $\Delta=0$ (pseudospin symmetry $\Sigma=0$ ) limitation $[16,17]$. Overmore, the solution of the Dirac equation can be easily reduced to it's nonrelativistic limit if one applies an appropriate map of parameters.

The rest of the paper is organized as follows. In section 2, we apply the NU method to solving the Schrödinger and Dirac equations with an exactly solvable isotonic oscillator to obtain the eigenvalues and eigenfunctions in a systematical way. We also compare the non-relativistic solution with the existing one obtained by applying the SUSY approach. In section 3, we make our summary and conclusions. 


\section{BOUND STATE SOLUTIONS}

\section{A. Schrödinger case}

We start with the one-dimensional single-particle Schrödinger equation $[18,19]$ :

$$
H_{\text {Isot }} \psi_{n}( \pm x)=\left[-\frac{\hbar^{2}}{2 M} \frac{d^{2}}{d( \pm x)^{2}}+U_{\text {Isot }}( \pm x)\right] \psi_{n}( \pm x)=E_{n} \psi_{n}( \pm x)
$$

The potential in Eq. (1) is invariant with respect to the inversion, i.e., $U_{\text {Isot }}(-x) \rightarrow U_{\text {Isot }}(x)$ as well as the hamiltonian $H_{\text {Isot }}$. Therefore, the Schrödinger equation should have even and odd solutions. The domain of the harmonic oscillator hamiltonian $H$ extends along the whole real axis $-\infty<x<\infty$, however, the partner $H_{\text {Isot }}$ exhibits a strong singularity at the origin. The space breaks up into two disjoint regions $(x \geq 0$ or $x \leq 0)$ without communication between them since the wave functions vanish at the regular singularity $x=0$ (i.e., $\psi_{n}(0)=0$ ) and at the irregular singularities $\pm \infty$ (i.e., $\psi_{n}( \pm \infty)=0$ ). In this respect, we should restrict the hamiltonian to the interval $(0, \infty)$, this is exactly the same situation that occurs in isotonic potential. In addition, if we set $x \rightarrow i x$, then (2) becomes

$$
\left[-\frac{\hbar^{2}}{2 M} \frac{d^{2}}{d x^{2}}+U_{\text {Isot }}(x)\right] \psi_{n}(i x)=-E_{n} \psi_{n}(i x)
$$

which is the original equation with the irrelevant change in the eigenvalues $E_{n} \rightarrow-E_{n}$. If we set $x \rightarrow i x$ in the isotonic wave function, we get perfectly good $\psi_{n}(i x)$ which can generate the superpotential $W(x)$ and other hamiltonians [6].

To solve Eq. (2) by NU method, we perform a straightforward algebra to reduce it into the following simple form:

$$
\psi_{n}^{\prime \prime}(x)+\left[\varepsilon_{n}-\beta^{2} x^{2}-\frac{\alpha}{x^{2}}\right] \psi_{n}(x)=0,
$$

with

$$
\varepsilon_{n}=\frac{2 M E_{n}}{\hbar^{2}}, \beta=\frac{M \omega}{\hbar}, \alpha=\frac{M g}{\hbar^{2}} \geq 0
$$

Let us restrict ourselves to the positive half-line $(x \geq 0)$ and in terms of new variable $s=x^{2}$ $(0 \leq s<\infty)$, we obtain

$$
\psi_{n}^{\prime \prime}(s)+\frac{1}{(2 s)} \psi_{n}^{\prime}(s)+\frac{1}{(2 s)^{2}}\left[-\beta^{2} s^{2}+\varepsilon_{n} s-\alpha\right] \psi_{n}(s)=0, \psi_{n}(0)=0,
$$


where we have used $\psi_{n}(x)=\psi_{n}(s)$. Now, if we compare the above equation with the following generalized hypergeometric-type equation with a parametrization of real variables $s=s(x)$ :

$$
\psi_{n}^{\prime \prime}(s)+\frac{\tilde{\tau}(s)}{\sigma(s)} \psi_{n}^{\prime}(s)+\frac{\tilde{\sigma}(s)}{\sigma^{2}(s)} \psi_{n}(s)=0,
$$

where

$$
\psi_{n}(s)=\Omega(s) y_{n}(s)
$$

and where $\sigma(s)$ and $\widetilde{\sigma}(s)$ are two polynomials, at most of second-degree, and $\widetilde{\tau}(s)$ is at most of first-degree polynomial, then it follows that:

$$
\widetilde{\tau}(s)=1, \sigma(s)=2 s, \tilde{\sigma}(s)=-\beta^{2} s^{2}+\varepsilon_{n} s-\alpha .
$$

To apply the NU method $[10,20]$, we calculate the function $\pi(s)$ defined by

$$
\begin{aligned}
\pi(s) & =\frac{\sigma^{\prime}(s)-\widetilde{\tau}(s)}{2} \pm \sqrt{\left[\frac{\sigma^{\prime}(s)-\widetilde{\tau}(s)}{2}\right]^{2}-\tilde{\sigma}(s)+k \sigma(s)} \\
& =\frac{1}{2}\left(1 \pm \sqrt{4 \beta^{2} s^{2}+4\left(2 k-\varepsilon_{n}\right) s+4 \alpha+1}\right)
\end{aligned}
$$

and also seek for a physical value of $k$ that makes the discriminant of the expression under square root, in the last equation, to become zero (i.e., $2 k=\varepsilon_{n} \pm \beta \sqrt{1+4 \alpha}, \alpha \geq-1 / 4$ ). Hence, there is no bound solution in the region $\alpha<-1 / 4$. The model becomes unphysical in the region $(-\infty,-1 / 4)$, since the spectrum is not bounded from below. Upon the substitution of the value of $k$ into the above equation, we obtain the following suitable solutions:

$$
\pi(s)=\frac{1}{2}(1+\sqrt{1+4 \alpha})-\beta s,
$$

and

$$
k=\frac{1}{2}\left(\varepsilon_{n}-\beta \sqrt{1+4 \alpha}\right) .
$$

With regard to Eqs. (8) and (10), we can calculate the function $\tau(s)=\widetilde{\tau}(s)+2 \pi(s)$, taking into consideration the bound state condition which has to be established when $\tau^{\prime}(z)<0$, as

$$
\tau(s)=2+\sqrt{1+4 \alpha}-2 \beta s \text { and } \tau^{\prime}(s)=-2 \beta<0 .
$$

According to the method, in order to find the energy equation from which one calculates the energy eigenvalues, we need to find the values of the parameters: $\lambda=k+\pi^{\prime}(s)$ and $\lambda=\lambda_{n}=-n \tau^{\prime}(s)-\frac{1}{2} n(n-1) \sigma^{\prime \prime}(s), n=0,1,2, \cdots$, as

$$
\lambda=\frac{1}{2}\left(\varepsilon_{n}-\beta \sqrt{1+4 \alpha}\right)-\beta \text { and } \lambda_{n}=2 n \beta, n=0,1,2, \cdots .
$$


Using the relation $\lambda=\lambda_{n}$ and the definitions of parameters in Eq. (4), one finds that the energy eigenvalues of the isotonic oscillator are

$$
E_{n, g}=\hbar \omega\left(2 n+1+\frac{1}{2} \sqrt{1+\frac{4 M g}{\hbar^{2}}}\right), n=0,1,2, \cdots,
$$

which is identical to the results of Ref. [21] (see p. 3). Thus, we have exactly solved the isotonic Hamiltonian (2). In the limit that $g \rightarrow 0$, the relation (14) reduces to $E_{n}=$ $\hbar \omega\left(2 n+\frac{3}{2}\right), n=0,1,2, \cdots$ which is identical to the $s$-wave solution of the three-dimensional Schrödinger equation with harmonic oscillator potential (cf. Eq. (34) when $l=0$ ).

Overmore, using the conventions of Ref. [9] (cf. Eqs. (41) and (43) therein), we may take $g=2$ (i.e., $m=1$ ) for easy of notation, the spectrum of Eq. (1) (in $\hbar=M=\omega=1$ units) reads as

$$
E_{n}=2 n+\frac{5}{2}, n=0,1,2, \cdots
$$

It is noticed that energy spectrum in the previous equation is half the spectrum of Eq. (41) in Ref. [9] (see Eq. (43) in [9]). The odd solutions under the inversion $x \rightarrow-x$ (the negative half-line, $x \leq 0$ ) has same energy spectrum as the even ones given in Eq. (14) due to the invariance of isotonic potential under this inversion. Hence, the energy relation in Eq. (14) holds for the whole domain $-\infty<x<\infty$.

Thus, all the other eigenenergies are given by

$$
E_{2 n}=E_{0}+2 n \omega, n=0,1,2, \cdots
$$

and the energy spectrum is equidistant since

$$
E_{2 n+2}=E_{2 n}+2 \omega
$$

Nevertheless, the height $\Delta E=2 \omega$ of the energy steps is twice that of the simple harmonic oscillator $U_{0}$. In fact, it seems as if half of of the states (those with an odd number of nodes) have disappeared.

Let us now turn to the calculations of the normalized wave function. The first part of the wave function in Eq. (7) is found through the relation [10,20]:

$$
\Omega(s)=\exp \left(\int \frac{\pi(s)}{\sigma(s)} d s\right)=s^{\frac{1}{4}+\frac{1}{2} \xi} \exp \left(-\frac{1}{2} \beta s\right),
$$

with

$$
\xi=\frac{1}{2} \sqrt{1+4 \alpha} \geq \frac{1}{2}
$$


and the calculation of the weight function is found through the relation,

$$
\rho(s)=\frac{1}{\sigma(s)} \exp \left(\int \frac{\tau(s)}{\sigma(s)} d s\right)=s^{\xi} \exp (-\beta s),
$$

leading to the calculation of the other part of the wave function; namely, $y_{n}(s)$ which is a hypergeometric type function whose polynomial solutions are given by the Rodrigues relation:

$$
y_{n}(s)=A_{n} \rho^{-1}(s) \frac{d^{n}}{d s^{n}}\left[\sigma^{n}(s) \rho(s)\right]=s^{-\xi} \exp (\beta s) \frac{d^{n}}{d s^{n}}\left(s^{n+\xi} \exp (-\beta s)\right)=L_{n}^{(\xi)}(\beta s),
$$

:where $L_{n}^{\alpha}(y)$ is the associated Laguerre polynomials. Therefore, the even solution of the wave function satisfying Eq. (7) is $[22,23]$

$$
\psi_{n}(x)=\sqrt{\frac{2 \beta^{1+\xi} n !}{\Gamma(n+\xi+1)}} x^{\frac{1}{2}+\xi} \exp \left(-\frac{1}{2} \beta x^{2}\right) L_{n}^{(\xi)}\left(\beta x^{2}\right), \quad \operatorname{Re} \xi>0 .
$$

It should be noticed that the change of $x \rightarrow i x$ in the eigenvalue equation (2) for the isotonic oscillator changes eigenvalues $E_{n} \rightarrow-E_{n}$. Then, if $\psi_{n}(x)$ is the eigenfunction corresponding to the eigenvalue $E_{n}$, then the eigenfunction $\psi_{n}(i x)$ will be normalizable only if $-E_{n}$ is in the point spectrum of this Hamiltonian. However, the $\psi_{n}(i x)$ would not be good wave functions if their spectrum $-E_{n}$ lies not within the Hamiltonian range, then $\psi_{n}(i x)$ is not normalizable eigenfunction. At first, it behaves like $\exp \left(\frac{1}{2} \beta x^{2}\right)$ at large $x$ but this is not relevant here as one writes the explicit form of $L_{n}^{(\xi)}\left(\beta x^{2}\right)$ for even $n$. On the other hand, the odd solutions for which the part of wave function corresponding to $-x$ have opposite signs and exist as

$$
\psi_{n}(-x)=\mathcal{N}_{n}(-x)^{\frac{1}{2}-\xi} \exp \left(-\frac{1}{2} \beta x^{2}\right) L_{n}^{(\xi)}\left(\beta x^{2}\right) .
$$

The two linearly independent solutions (wave functions) given by Eqs. (22) and (23) for the even and odd solutions, respectively, need to be normalizable in the range $(0, \infty)$. However, the odd solution is not normalizable in the region $(0, \infty)$ as one can see in Eq. (23). Indeed, the operator in (3) is not essentially self-adjoint for $-1 / 4 \leq \alpha<3 / 4$ and its most general square-integrable solution behaves near the singularity as a linear combination of $x^{1 / 2+\xi}$ and $x^{1 / 2-\xi}$ (See Eqs. (22) and (23)).[1] In this respect, we clarify this point by analyzing the behaviour of the isotonic potential (1) in terms of the parameter $\alpha$ [24,25], three different regions appear, namely,

[1] We would like to thank one of the referees for drawing our attention to this point. 
- In the range $\alpha \in(-\infty,-1 / 4)$ the model becomes unphysical since the spectrum is not bounded from below (see Figure 1) [24].

- When $\alpha \in(-1 / 4,3 / 4)$, the singularity is not strong enough to make the wave functions (22) and (23) vanish at $x=0$. Indeed in this region both linearly independent solutions (wave functions) are normalizable since $\xi=0$ and 1 . This is the reason why it is necessary to select, from the continuous family of self-adjoint extensions by the differential operator, the self-adjoint extensions which correctly describes the physical system under consideration [24]. The wave functions pass across the singularity point $x=0$ and the model extends itself again along the entire region, that is; $(-\infty, \infty)$.

- Physically, if we consider the range $\alpha \in(3 / 4, \infty)$, the singularity acts as an impentrable barrier, thus dividing the space into two independent regions, that is; $x \leq 0$ and $x>0$. The wave functions must vanish at $x=0$ which provides an absolute lack of communication between the two regions of space (i.e., the negative and positive half-lines) and the wave functions (22) and (23) in this case become normalizable $[24,25]$.

Alternatively, notice the Laguerre polynomial can be expressed in terms of the Kummer confluent hypergeometric functions as [23]

$$
\left.L_{n}^{p}(z)=\frac{(p+n) !}{p ! n !}{ }_{1} F_{1}(-n ; p+1 ; z)\right) \text {, }
$$

where

$$
{ }_{1} F_{1}(a ; b ; z)=1+\frac{a}{b} z+\frac{a(1+a)}{2 b(1+b)} z^{2}+\frac{a(1+a)(2+a)}{6 b(1+b)(2+b)} z^{3}+O[z]^{4} .
$$

Using the notations of other authors $(\hbar=M=1)[21]$ and putting $\beta=\omega$ and $\xi=m+1 / 2$, the even wave function solution in Eq. (22) becomes [23]

$$
\begin{gathered}
\psi_{n}(x)=N_{n} x^{1+m} \exp \left(-\frac{1}{2} \omega x^{2}\right){ }_{1} F_{1}\left(-n ; m+\frac{3}{2} ; \omega x^{2}\right), n=0,2,4, \cdots, \\
N_{n}=\frac{1}{\Gamma\left(m+\frac{3}{2}\right)} \sqrt{\frac{2 \omega^{m+\frac{3}{2}} \Gamma\left(n+m+\frac{3}{2}\right)}{n !}},
\end{gathered}
$$

which is identical to Eq. (42) in Ref. [9] when we set $m=1$ so that the isotonic potential given by Eq. (41) in [9] is twice the potential (1) in the present work. On the other hand, 
the odd solution for which the part of wave function corresponding to $-x$ can be expressed as

$$
\psi_{n}(-x)=(-1)^{1+m} \psi_{n}(x), n=1,3,5, \cdots .
$$

Hence, if we take $m=0,2,4, \cdots$ (even real integer), we find the wave function $\psi_{n}(-x)$ being an odd (antisymmetric) function of $x$ [i.e., $\left.\psi_{n}(-x)=-\psi_{n}(x)\right]$. Overmore, if we take $m=1,3,5, \cdots$ (odd real integer), we find the wave function corresponding to negative values of $x$ is identical to the wave function corresponding to positive values, i.e., $\psi_{n}(-x)$ being an even function (symmetric) with $\psi_{n}(-x)=\psi_{n}(x)$. In case if $m$ is a real number but not integer yielding a complex wave function in the negative half-line which is not normalizable.

On the other hand, the energy levels of the one-dimensional Schrödinger equation for the harmonic oscillator $U_{0}(x)[1]$ :

$$
E_{n}=\left(n+\frac{1}{2}\right) \hbar \omega, n=0,1,2, \cdots
$$

and the well-known wave functions [26]

$$
\phi_{n}(x)=\left[\frac{1}{2^{n} n !} \sqrt{\frac{\beta}{\pi}}\right]^{1 / 2} H_{n}\left(\beta x^{2}\right) \exp \left(-\frac{1}{2} \beta x^{2}\right), \beta=\frac{M \omega}{\hbar},
$$

where $H_{n}(y)=(-1)^{n} \exp \left(y^{2}\right) \frac{d^{n}}{d y^{n}} \exp \left(-y^{2}\right)$ represent Hermite polynomials.

For further discussions on the isotonic potential, we present the energy eigenvalues and the corresponding wave functions of the lowest three states,

$$
\begin{gathered}
E_{0}=\omega\left(\frac{3}{2}+m\right), \\
\psi_{0}(x)=N_{0} x^{1+m} \exp \left(-\frac{1}{2} \omega x^{2}\right), \\
E_{1}=\left(\frac{5}{2}+m\right) \omega, \\
\psi_{1}(x)=N_{1} x^{1+m} \exp \left(-\frac{1}{2} \omega x^{2}\right)\left(1-\frac{2 \omega}{(2 m+3)} x^{2}\right),
\end{gathered}
$$

and

$$
\begin{gathered}
E_{2}=\left(\frac{7}{2}+m\right) \omega \\
\psi_{2}(x)=N_{2} x^{1+m} \exp \left(-\frac{1}{2} \omega x^{2}\right)\left(1-\frac{4 \omega}{(2 m+3)} x^{2}+\frac{4 \omega^{2}}{(2 m+3)(2 m+5)} x^{4}\right),
\end{gathered}
$$


respectively and the normalization factors are calculated as

$$
N_{0}=\sqrt{\frac{2 \omega^{m+\frac{3}{2}}}{\Gamma\left(m+\frac{3}{2}\right)}}, N_{2}=(-1)^{1+m} \sqrt{\frac{\omega^{m+\frac{3}{2}}(2 m+3)}{\Gamma\left(m+\frac{3}{2}\right)}}, N_{2} \sqrt{\frac{\omega^{m+\frac{3}{2}}(2 m+5)(2 m+3)}{4 \Gamma\left(m+\frac{3}{2}\right)}} .
$$

The isotonic ground state wave function, $\psi_{0}(x)$ in $(30 \mathrm{~b})$ is compared with the corresponding harmonic oscillator wave function, $\phi_{0}(x)$ in Eq. (29) in Figure 2. Further, the isotonic first two excited wave functions, $\psi_{1}(x)$ and $\psi_{2}(x)$ in Eqs. (31b) and (32b) are compared with the corresponding harmonic oscillator wave functions, $\phi_{1}(x)$ and $\phi_{2}(x)$ in Eq. (29) in Figures 3 and 4 , respectively.

On the other hand, the solution of the three-dimensional Schrödinger equation with any arbitrary quantum number $l$ (i.e., harmonic oscillator combined with centrifugal barrier potential) provides us

$$
E_{n, l}=\hbar \omega\left(2 n+l+\frac{3}{2}\right), n, l=0,1,2, \cdots
$$

and the corresponding wave functions are given by

$$
\psi_{n, l}(r, \theta, \varphi)=\sqrt{\left(\frac{\beta}{\pi}\right)^{1 / 2} \frac{2^{n+2 l+3} n !(2 \beta)^{l}}{(2 n+2 l+1) ! !}} r^{l} \exp \left(-\frac{1}{2} \beta r^{2}\right) L_{n}^{(l+1 / 2)}\left(\beta r^{2}\right) Y_{l, m}(\theta, \varphi)
$$

where $L_{n}^{(l+1 / 2)}\left(\beta r^{2}\right)$ is the associated Laguerre polynomial, and $Y_{l, m}(\theta, \varphi)$ is the angular part of the wave functions. The order $n$ of the polynomial is a non-negative integer. Thus, the exact solution of the isotonic oscillator in Eq. (14) in one-dimension is equivalent to the solution of the harmonic oscillator $U_{0}(r)$ combined with the centrifugal barrier potential $l(l+1) / r^{2}, r \in(0, \infty)$, in three-dimensions given in Eq. (34) when we take $\hbar=M=1$ and $g=m(m+1)$, where $g$ is a real number. That is, $E_{n, m}=\hbar \omega\left(n_{1}+\frac{3}{2}\right)$ is equivalent to $E_{n, l}=\hbar \omega\left(n_{2}+\frac{3}{2}\right)$, where we have defined $n_{1}=2 n+m$ and $n_{2}=2 n+l(m \leftrightarrow l)$ for which the solutions are defined for positive half-line $[x \in(0, \infty) \leftrightarrow r \in(0, \infty)]$.

\section{B. Dirac Case}

We start by writting the two radial coupled Dirac equations for the upper and lower (i.e., $F_{n, \kappa}(r)$ and $G_{n, \kappa}(r)$, respectively) spinor components [26,27]:

$$
\left(\frac{d}{d r}+\frac{\kappa}{r}\right) F_{n, \kappa}(r)=\left(M c^{2}+E_{n \kappa}-\Delta\right) G_{n, \kappa}(r)
$$




$$
\left(\frac{d}{d r}-\frac{\kappa}{r}\right) G_{n, \kappa}(r)=\left(M c^{2}-E_{n \kappa}+\Sigma\right) F_{n, \kappa}(r)
$$

where $\Delta=V-S$ and $\Sigma=V+S$ are the difference and sum potentials, respectively and are expressed in terms of vector $(V)$ and scalar $(S)$ potentials. In addition, $c \approx 137$ is the velocity of light.

In the presence of spin symmetry ( i.e., $\Delta=C_{s}$ ), one gets a second-order differential equation satisfying the upper-spinor component [17,28-31]

$$
F_{n \kappa}^{\prime \prime}(r)-\left(\frac{\kappa(\kappa+1)}{r^{2}}+A_{s}^{2}+\gamma \Sigma\right) F_{n \kappa}(r)=0,
$$

where

$$
A_{s}^{2}=\gamma\left(M c^{2}-E_{n \kappa}\right), \gamma=\frac{1}{\hbar^{2} c^{2}}\left(M c^{2}+E_{n \kappa}-C_{s}\right)>0
$$

and $\kappa(\kappa+1)=l(l+1), \kappa=l$ for $\kappa<0$ and $\kappa=-(l+1)$ for $\kappa>0$. The spin symmetry energy eigenvalues depend on $n$ and $\kappa$, i.e., $E_{n \kappa}=E(n, \kappa(\kappa+1))$. For $l \neq 0$, the states with $j=l \pm 1 / 2$ are degenerate. Further, the lower-spinor component can be obtained from Eq. (36a) as

$$
G_{n \kappa}(r)=\frac{1}{M c^{2}+E_{n \kappa}-C_{s}}\left(\frac{d}{d r}+\frac{\kappa}{r}\right) F_{n \kappa}(r),
$$

where $E_{n \kappa} \neq-M c^{2}$, i.e., only real positive energy states exist when $C_{s}=0$ (exact spin symmetric case).

On the other hand, under the pseudospin symmetry ( i.e., $\Sigma=C_{p s}$ ), one obtains a second-order differential equation satisfying the lower-spinor component,

$$
G_{n \kappa}^{\prime \prime}(r)-\left(\frac{\kappa(\kappa-1)}{r^{2}}+A_{p s}^{2}-\widetilde{\gamma} \Delta\right) G_{n \kappa}(r)=0,
$$

where

$$
A_{p s}^{2}=\widetilde{\gamma}\left(M c^{2}+E_{n \kappa}\right), \widetilde{\gamma}=\frac{1}{\hbar^{2} c^{2}}\left(M c^{2}-E_{n \kappa}+C_{p s}\right)
$$

and the upper-spinor component $F_{n \kappa}(r)$ is obtained from Eq. (36b) as

$$
F_{n \kappa}(r)=\frac{1}{M c^{2}-E_{n \kappa}+C_{p s}}\left(\frac{d}{d r}-\frac{\kappa}{r}\right) G_{n \kappa}(r),
$$

where $E_{n \kappa} \neq M c^{2}$, i.e., only real negative energy states exist when $C_{p s}=0$ (exact pseudospin symmetric case). From the above equations, the energy eigenvalues depend on the quantum numbers $n$ and $\kappa$, and also the pseudo-orbital angular quantum number $\tilde{l}$ according to $\kappa(\kappa-1)=\tilde{l}(\tilde{l}+1)$, which implies that $j=\tilde{l} \pm 1 / 2$ are degenerate for $\tilde{l} \neq 0$. The quantum 
condition for bound states demands the finiteness of the solution at infinity and at the origin points, i.e., $F_{n \kappa}(0)=G_{n \kappa}(0)=0$ and $F_{n \kappa}(\infty)=G_{n \kappa}(\infty)=0$.

Let us now study the isotonic potential (1) in the context of spin and pseudospin symmetric Dirac equations. It is well-known that Eqs. (37) and (40) can be solved exactly for any $\kappa$ with the spin-orbit (pseudospin-orbit) centrifugal (pseudo centrifugal) potential term. However, we shall study these equations for the $s$-wave case $(\kappa= \pm 1)$ for the sake of comparison with the nonrelativistic case since $m(m+1) / x^{2}$ in the isotonic potential has the same behaviour as $\kappa(\kappa \pm 1) / r^{2}$ in Eqs. (37) and (40).

\section{Spin symmetry limit}

This symmetry arises from the near equality in magnitude of an attractive scalar, $S$, and repulsive vector, $V$, relativistic mean field, $S \sim V$ in which the nucleon move [12]. Therefore, we simply take the sum potential equal to the isotonic potential model, i.e.,

$$
\Sigma=U_{\text {Isot }}(x)=\frac{1}{2} M \omega^{2} x^{2}+\frac{1}{2} \frac{g}{x^{2}} .
$$

In the last equation, the choice of $\Sigma=2 V \rightarrow U_{\text {Isot }}(x)$ as stated in Ref. [26] allows one to reduce it into its non-relativistic limit under appropriate choice of parameter transformations. Further, we take $\kappa=-1(l=0)$ and in terms of new variable $s=x^{2}$ (positive half-plane $x \geq 0$ ), Eq. (37) becomes

$$
F_{n,-1}^{\prime \prime}(s)+\frac{1}{(2 s)} F_{n,-1}^{\prime}(s)+\frac{1}{(2 s)^{2}}\left[-\nu^{2} s^{2}-A_{s}^{2} s-\beta\right] F_{n,-1}(s)=0,
$$

where

$$
\beta=\frac{1}{2} g \gamma \text { and } \nu=\sqrt{\frac{1}{2} M \omega^{2} \gamma} .
$$

The quantum condition is obtained from the finiteness of the solution at infinity and at the origin point. We apply the NU method following the same steps of solution in previous section to obtain the expressions:

$$
\widetilde{\tau}(s)=1, \sigma(s)=2 s, \tilde{\sigma}(s)=-\nu^{2} s^{2}-A_{s}^{2} s-\beta .
$$

It follows that the functions required by the method for $\pi(s), k$ and $\tau(s)$ take the suitable forms:

$$
\pi(s)=-\nu s+\frac{1}{2}(1+\sqrt{1+4 \beta})
$$




$$
k=-\frac{1}{2}\left(A_{s}^{2}+\nu \sqrt{1+4 \beta}\right)
$$

and

$$
\tau(s)=2+\sqrt{1+4 \beta}-2 \nu s \text { and } \tau^{\prime}(s)=-2 \nu<0,
$$

respectively, with prime denotes the derivative with respect to $s$. Also, the parameters $\lambda$ and $\lambda_{n}$ take the forms:

$$
\lambda=-\frac{1}{2}\left(A_{s}^{2}+\nu \sqrt{1+4 \beta}\right)-\nu, \text { and } \lambda_{n}=2 n \nu .
$$

Using the condition $\lambda=\lambda_{n}$ followed by simple algebra, we obtain the following transcendental energy equation,

$$
\left(E_{n,-1}-M c^{2}\right) \sqrt{M c^{2}+E_{n,-1}-C_{s}}=\hbar c \omega \sqrt{2 M}\left(2 n+1+\frac{1}{2} \sqrt{\frac{2 g}{\hbar^{2} c^{2}}\left(M c^{2}+E_{n,-1}-C_{s}\right)+1}\right),
$$

where $n=0,1,2,3, \cdots$ and $E_{n,-1} \geq C_{s}-M c^{2}$. One can compute the energy eigenvalues by choosing suitable parameters in the symmetric potential. Hence, Eq. (51) shows the energy eigenvalues $E_{n}$ dependence on $n$ and $C_{s}$ as well as on the parameters $\omega$ and $M$.

Therefore, using Eq. (51), we compute some energy levels for several values of $n$ (in units $\hbar=c=1$ ). In the presence spin symmetric limit, Table 1 gives some numerical results by taking the following parameters values: $M=\omega=1.0 \mathrm{fm}^{-1}, C_{s}=0 \mathrm{fm}^{-1}$ (exact symmetric case) and $C_{s}=2.0 \mathrm{fm}^{-1}$ (non exact symmetric case). Moreover, the strength of the centripetal barrier term is set up to some arbitrarily chosen values: $g=0.5,2$ and 6 corresponding to $m=0.3660254,1$ and 2, respectively. For the values $g=2$ and 6 , the singularity acts as impenetrable barrier, thus deviding the space into two independent regions, the negative half-line and the positive half-line.

Dirac equation which in the limit of a non-relativistic and spinless particle transforms into Schrödinger equation for the isotonic potential (1) is constructed as follows. In the exact spin symmetry, we set $C_{s}=0$ and apply appropriate transformations given by $\left(M c^{2}+E_{n,-1}\right) / \hbar^{2} c^{2} \simeq 2 M / \hbar^{2}$ and $E_{n,-1}-M c^{2} \simeq E_{n}$, we finally obtain the Schrödinger solution in (14).

Let us now turn to the calculations of the corresponding wave functions for this system. We obtain the first part $\phi(s)$ of the wave function (7) and the weight function $\rho(s)$ as

$$
\Omega(s)=s^{\frac{1}{4} 1+\frac{1}{2} \zeta} \exp \left(-\frac{1}{2} \nu s\right)
$$


where

$$
\zeta=\frac{1}{2} \sqrt{1+2 g \gamma}, g=m(m+1)
$$

and

$$
\rho(s)=s^{\zeta} \exp (-\nu s) .
$$

Hence, the second part $y_{n}(s)$ of the wave function (7) can be obtained from the weight function as

$$
y_{n}(s) \sim L_{n}^{(\zeta)}(\nu s)
$$

Finally, we find the normalized wave function satisfying Eq. (37) as

$$
F_{n,-1}(x)=\sqrt{\frac{2 \nu^{1+\zeta} n !}{\Gamma(n+\zeta+1)}} x^{\frac{1}{2}+\zeta} \exp \left(-\frac{1}{2} \nu x^{2}\right) L_{n}^{(\zeta)}\left(\nu x^{2}\right) .
$$

In addition, the corresponding lower-spinor component wave function $G_{n,-1}(x)$ is found from the solution of Eq. (39) as

$$
\begin{aligned}
G_{n,-1}(x) & =\frac{1}{\left(M c^{2}+E_{n,-1}-C_{s}\right)} \sqrt{\frac{2 \nu^{1+\zeta} n !}{\Gamma(n+\zeta+1)}} x^{\frac{1}{2}+\zeta} \exp \left(-\frac{1}{2} \nu x^{2}\right) \\
& \times\left[\left(\frac{-1+2 \zeta}{2 x}-\nu x\right) L_{n}^{(\zeta)}\left(\nu x^{2}\right)+\frac{d L_{n}^{(\zeta)}\left(\nu x^{2}\right)}{d x}\right] .
\end{aligned}
$$

Let us remark that the obtained results of the $s$-wave $(\kappa=-1)$ of the spin-symmetric Dirac equation with isotonic oscillator agree with the results of the three dimensional Dirac equation with harmonic oscillator potential combined with the centrifugal barrier term $\kappa(\kappa+1) / r^{2}$ if we make the parameter change $m \leftrightarrow \kappa$ since the positive half-line $x \in(0, \infty)$ in the first is also equivalent to $r \in(0, \infty)$ in the second. This is apparent because the centripetal barrier potential $m(m+1) / x^{2}$ in the isotonic oscillator is equivalent to the centrifugal term $\kappa(\kappa+1) / r^{2}$ in Eq. (37).

On the other hand, the Klein-Gordon solution for the isotonic potential (in relativistic $\hbar=c=1$ units) can be obtained from the exact spin-symmetric case, $V=S, C_{s}=0$. Hence, the energy equation can be obtained from Eq. (51) as

$$
\left(E_{n,-1}^{2}-M^{2}\right)\left(E_{n,-1}-M\right)=2 M \omega^{2}\left(2 n+1+\frac{1}{2} \sqrt{1+2 g\left(M c^{2}+E_{n,-1}\right)}\right)^{2},
$$

and the wave function from Eq. (56) as

$$
F_{n,-1}(x)=\sqrt{\frac{2 n !(\epsilon)^{\frac{1}{2}\left(1+\lambda_{0}\right)}}{\Gamma\left(n+\lambda_{0}+1\right)}} x^{\frac{1}{2}+\lambda_{0}} \exp \left(-\frac{1}{2} \epsilon x^{2}\right) L_{n}^{\left(\lambda_{0}\right)}\left(\epsilon x^{2}\right),
$$




$$
\epsilon=\sqrt{\frac{1}{2} M \omega^{2}\left(M+E_{n,-1}\right)}, \quad \lambda_{0}=\frac{1}{2} \sqrt{1+2 g\left(M+E_{n,-1}\right)} .
$$

\section{Pseudospin symmetry limit}

The exact pseudospin symmetry occurs when $S \sim-V$ or $\Sigma=C_{p s}=$ constant $[12,16]$ and the quality of the pseudospin approximation in real nuclei is connected with the competition between the pseudo-centrifugal barrier and the pseudospin-orbital potential [32]. Therefore, we take the difference potential in Eq. (40) as the isotonic potential model, i.e.,

$$
\Delta=U_{\text {Isot }}(x)=\frac{1}{2} M \omega^{2} x^{2}+\frac{1}{2} \frac{g}{x^{2}}
$$

In the pseudospin symmetry, the eigenstates with with $\widetilde{j}=\tilde{l} \pm \frac{1}{2}$ are degenerate for $\tilde{l} \neq 0$. For the $s$-wave case $(\kappa=1)$ and in terms of the variable $s=x^{2}$, Eq. (40) reduces to a simple form

$$
G_{n, 1}^{\prime \prime}(s)+\frac{1}{(2 s)} G_{n, 1}^{\prime}(s)+\frac{1}{(2 s)^{2}}\left[\widetilde{\nu}^{2} s^{2}-A_{p s}^{2} s+\widetilde{\beta}\right] G_{n, 1}(s)=0
$$

where

$$
\widetilde{\beta}=\frac{1}{2} g \widetilde{\gamma} \text { and } \widetilde{\nu}=\sqrt{\frac{1}{2} M \omega^{2} \widetilde{\gamma}}
$$

To avoid repetition in the solution of Eq. (61), a first inspection for the relationship between the present set of parameters $\left(A_{p s}^{2}, \widetilde{\beta}, \widetilde{\nu}\right)$ and the previous set $\left(A_{s}^{2}, \beta, \nu\right)$ provides that the energy solution for pseudospin symmetry can be similarly found directly from those of the previous energy solutions for spin symmetry using the following parameters map [33]:

$$
\begin{gathered}
F_{n,-1}(s) \leftrightarrow G_{n, 1}(s), E_{n,-1} \rightarrow-E_{n, 1}, C_{s} \rightarrow-C_{p s}, A_{s}^{2} \rightarrow A_{p s}^{2} \\
U(s) \rightarrow-U(s)\left(\nu^{2} \rightarrow-\widetilde{\nu}^{2}, \beta \rightarrow-\widetilde{\beta} \text { or } \omega \rightarrow j \omega, g \rightarrow-g\right), j=\sqrt{-1},
\end{gathered}
$$

from which trivial calculus gives us the transcendental energy equation:

$$
\left(E_{n, 1}+M c^{2}\right) \sqrt{E_{n, 1}-M c^{2}-C_{p s}}=\hbar c \omega \sqrt{2 M}(2 n+1+\widetilde{\zeta})
$$

with

$$
\widetilde{\zeta}=\frac{1}{2} \sqrt{1+\frac{2 g}{\hbar^{2} c^{2}}\left(E_{n, 1}-M c^{2}-C_{p s}\right)} .
$$

where $n=0,1,2,3, \cdots$ and $E_{n, 1} \geq M c^{2}+C_{p s}$ is the main condition for the real bound state solutions. 
Now the normalized lower-spinor component wavefunctions of the isotonic oscillator are given by

$$
F_{n, 1}(x)=\sqrt{\frac{2(i \widetilde{\nu})^{1+\widetilde{\zeta}} n !}{\Gamma(n+\widetilde{\zeta}+1)}} x^{\frac{1}{2}+\widetilde{\zeta}} \exp \left(-\frac{1}{2} \widetilde{\nu} x^{2}\right) L_{n}^{(\widetilde{\zeta})}\left(i \widetilde{\nu} x^{2}\right) .
$$

Therefore, using Eq. (64), we compute some energy levels for several values of $n$. In the pseudospin symmetric limit, Table 2 gives some numerical results by taking the following parameters values: $M=\omega=1.0 \mathrm{fm}^{-1}, C_{s}=0 \mathrm{fm}^{-1}$ (exact symmetric case) and $C_{s}=-2.0$ $\mathrm{fm}^{-1},-13.0 \mathrm{fm}^{-1}$ (non exact symmetric case). In addition, the strength of the centripetal barrier term is set up to the following arbitrarily chosen values: $g=0.5,2$, and 6 .

\section{CONCLUSIONS AND OUTLOOK}

In this work, qualitative data were obtained on the modifications of spectrum energy on a nonrelativistic and relativistic particle confined by isotonic oscillator field of specific strength $g$. The spin and pseudospin symmetry in relativistic isotonic oscillator are investigated systemically by solving the Dirac equation with scalar and vector radial potentials by applying the NU method. In one-dimensional isotonic oscillator, we have obtained the exact solutions in closed form for the energy spectrum and the wave functions, which are equivalent to solving the three-dimensional harmonic oscillator problem. The isotonic oscillator is an isospectral to harmonic oscillator. Also, the energy steps are twice that of the simple harmonic oscillator. The resulting solutions of the wave functions are written in terms of the associated Laguerre polynomials $L_{n}^{\alpha}(z)$ (confluent hypergeometric functions $\left.M(a, b, z)={ }_{1} F_{1}(a ; b ; z)\right)$ and the wave function for states $n=0,1$ and 2 are found to have the same shape as the harmonic oscillator as shown in Figures 2, 3 and 4. The case where $\mathrm{n}$ is even appears to be the most interesting, since all generated wave functions are normalizable. However, when $n$ is odd, half of the generated wave functions must be removed as they are not normalizable.

In the relativistic case, it is found that the solutions when $\Delta=0 \rightarrow S=V$ (i.e., exact symmetric case, $\left.C_{s}=0\right)$ or $\Sigma=0 \rightarrow S=-V$ (i.e., exact pseudosymmetric case, $C_{p s}=0$ ) are identical to the Klein-Gordon solutions. Besides, they can be readily reduced to the expected nonrelativistic limit when appropriate mapping transformations of parameters are made. In the numerical work, the relativistic energy spectrum for the spin and pseudospin 
symmetries are given in Tables 1 and 2, respectively. It is noticed that the parameters $g$, $M, C_{s}$ and $C_{p s}$ should be adjusted to provide us real solutions for the energy eigenvalues and eigenfunctions.

Finally, let us also mention that the isotonic oscillator possesses a remarkable property. The change of $x \rightarrow i x$ in the wave equation resulting in the change of eigenvalues $E_{n} \rightarrow$

$-E_{n}$. Then, if $\psi_{n}(x)$ is the eigenfunction corresponding to the eigenvalue $E_{n}$, then the eigenfunction $\psi_{n}(i x)$ will be normalizable only if $-E_{n}$ is in the point spectrum of this Hamiltonian. So the isotonic oscillator wave function with the change $x \rightarrow i x$ would be a good wave function as they are normalizable $\left(-E_{n} \in\langle n|H| n\rangle\right)$ or would not be good if they are not normalizable $\left(-E_{n} \notin\langle n|H| n\rangle\right)$. The eigenfunction $\psi_{n}(i x)$ can be used to generate new operators in the supersymmetric quantum mechanics [6,7]. This remains as an open question that deserves to be studied.

\section{Acknowledgments}

We acknowledge the kind referee(s) for the inavaluable suggestions that helped us to improve this paper greatly. 
[1] S. Flügge, Practical Quantum mechanics I (Berlin, Springer, 1994).

[2] M.A. Olshanetski and A.M. Perelomov, Phys. Rep. 94, 313 (1983).

[3] R. Dutt, A. Gangopadhyaya, C. Rasinariu and U. Sukhatme, J. Phys. A: Math. Gen. 34, 4129 (2001).

[4] R. Koc, M. Koca and E. Körcük, J. Phys. A. Math. Gen. 35, L527 (2002).

[5] J.F. Cariñena, M.F. Rañada and M. Santander, Ann. Phys. 322, 434 (2007).

[6] J.M. Fellows and R.A. Smith, J. Phys. A: Math. Theor. 42, 335303 (2009).

[7] F. Cooper, A. Khare and U. Sukhatme, Supersymmetry in Quantum Mechanics (Singapore, World Scientific, 2001).

[8] L. Infeld and T.E. Hull, Rev. Mod. Phys. 23, 21 (1951).

[9] J. Casahorrán, Physica A 217, 429 (1995).

[10] A.F. Nikiforov and V.B. Uvarov, Special Functions of Mathematical Physics (Birkhäuser, Basel, 1988).

[11] W. Greiner, Relativistic Quantum Mechanics (Springer, Verlag, 1981).

[12] J.N. Ginocchio, Phys. Rev. C 69, 034318 (2004); J.N. Ginocchio, Phys. Rev. Lett. 78, 436 (1997); J.N. Ginocchio, Phys. Rep. 414, 165 (2005).

[13] R. Lisboa, M. Malheiro, A.S. de Castro, P. Alberto and M. Fiolhais, Phys. Rev. C 69, 024319 (2004).

[14] R. Lisboa, M. Malheiro, A.S. de Castro, P. Alberto and M. Fiolhais, Int. J. Mod. Phys. D 13, 1447 (2004).

[15] J.N. Ginocchio, Phys. Rep. 315, 231 (1999).

[16] J. Meng, K. Sugawara-Tanabe, S. Yamaji and A. Arima, Phys. Rev. C 59, 154 (1999).

[17] S.M. Ikhdair and R. Sever, Cent. Eur. J. Phys. 8 (4), 652 (2010).

[18] L. I. Schiff, Quantum Mechanics 3rd edn. (McGraw-Hill Book Co., New York, 1968).

[19] L. D. Landau and E. M. Lifshitz, Quantum Mechanics, Non-relativistic Theory, 3rd edn. (Pergamon, New York, 1977).

[20] S.M. Ikhdair, Eur. Phys. J. A 40 (2), 143 (2009).

[21] J.F. Cariñena, A.M. Perelomov, M.F. Rañada and M. Santander, J. Phys. A: Math. Theor. 
41, 085301 (2008).

[22] O. Aydoğdu and R. Sever, Phys. Scr. 80, 015001 (2009).

[23] I.S. Gradshtein and I.M. Ryzhik, Tables and integrals, series and products (New York, Academic, 1969); M. Abramowitz and I.A. Stegun, Handbook of Mathematical Functions (New York, Dover Publication, 1972).

[24] M. Reed and B. Simon, Methods of Modern Mathematical Physics II: Fourier Analysis, SelfAdjointness (Academic Press, New York, 1975).

[25] L. Lathouwers, J. Math. Phys. 16, 1393 (1975).

[26] P.A.M. Dirac, The Principles of quantum Mechanics (Oxford Univ. Press, Oxford, 1958).

[27] W. Greiner, Relativistic Quantum Mechanics (Springer, Verlag, 1981).

[28] S.M. Ikhdair, J. Math. Phys. 51 (2), 023525 (2010).

[29] S.M. Ikhdair, J. Math. Phys. 52 (5), 052303 (2011).

[30] S.M. Ikhdair and R. Sever, J. Phys.: Math. Theor. 44, 345301 (2011).

[31] S.M. Ikhdair, C. Berkdemir and R. Sever, Appl. Math. Comput. 217, 9019 (2011)

[32] J.-Y. Guo, X.-Z. Fang and F.-X. Xu, Nuclear Phys. A 757, 411 (2005).

[33] C. Berkdemir and Y.-F. Cheng, Phys. Scr. 79, 035003 (2009). 
FIG. 1: Behaviour of the isotonic oscillator potential (continuous line) and the harmonic oscillator potential (dash line).

FIG. 2: Behaviour of the ground state wave function $\psi_{n=0, m=1}(x)$ of the isotonic oscillator (continuous line) and the corresponding wave function $\phi_{0}(x)$ of the harmonic oscillator (dash line).

FIG. 4: Behaviour of the second excited wave function $\psi_{n=2, m=1}(x)$ of the isotonic oscillator (continuous line) and the corresponding wave function $\phi_{2}(x)$ of the harmonic oscillator (dash line).

FIG. 3: Behaviour of the first excited wave function $\psi_{n=1, m=1}(x)$ of the isotonic oscillator (continuous line) and the corresponding wave function $\phi_{1}(x)$ of the harmonic oscillator (dash line). 
TABLE I: The spin symmetric bound state energy eigenvalues (in $\mathrm{fm}^{-1}$ ), for several values of $n$ with parameter values $M=1.0 \mathrm{fm}^{-1}$ and $\omega=1.0 \mathrm{fm}^{-1}$.

\begin{tabular}{llllll}
\hline \multicolumn{3}{c}{$C_{s}=0 \mathrm{fm}^{-1} a$} & & \multicolumn{3}{c}{$C_{s}=2.0 \mathrm{fm}^{-1}$} \\
$n / E_{n}$ & $g=0.5(m \approx 0.366)$ & $g=2(m=1)$ & $g=6(m=2)$ & $g=2(m=1)$ & $g=6(m=2)$ \\
\hline 0 & 2.5509860 & 3.1503636 & 4.0959121 & 3.3991120 & 4.2634174 \\
1 & 3.7292142 & 4.2915849 & 5.1735045 & 4.6747397 & 5.4772542 \\
2 & 4.7223578 & 5.2667833 & 6.1147629 & 5.7095838 & 6.4867680 \\
3 & 5.6093599 & 6.1428129 & 6.9690531 & 6.6208542 & 7.3835758 \\
4 & 6.4244044 & 6.9503157 & 7.7611866 & 7.4521361 & 8.2052891 \\
5 & 7.1861562 & 7.7065008 & 8.5058073 & 8.2256717 & 8.9719327 \\
6 & 7.9061955 & 8.4222280 & 9.2124501 & 8.9547327 & 9.6957461 \\
7 & 8.5923225 & 9.1048960 & 9.8877527 & 9.6480343 & 10.3848919 \\
8 & 9.2501029 & 9.7598277 & 10.5365663 & 10.3116853 & 11.0451537 \\
9 & 9.8836823 & 10.3910117 & 11.1625702 & 10.9501754 & 11.6808166 \\
10 & 10.4962522 & 11.0015335 & 11.7686371 & 11.5669263 & 12.2951658 \\
\hline
\end{tabular}

${ }^{a}$ Exact spin symmetric limit. 
TABLE II: The pseudospin symmetric bound state energy eigenvalues (in $\mathrm{fm}^{-1}$ ), for several values of $n$ with parameter values $M=1.0 \mathrm{fm}^{-1}$ and $\omega=1.0 \mathrm{fm}^{-1}$.

\begin{tabular}{lllllllll}
\hline \multicolumn{3}{c}{$C_{p s}=0^{a}$} & & \multicolumn{3}{c}{$C_{p s}=-2.0$} & \multicolumn{3}{c}{$C_{p s}=-13.0$} \\
$n / E_{n} g=0.5$ & $g=2$ & $g=6$ & $g=0.5$ & $g=2$ & $g=6$ & $g=2$ & $g=6$ \\
\hline 0 & 1.7353829 & 1.9975105 & 2.6220370 & 0.8996794 & 1.3991120 & 2.2634174 & 0.8228652 & 1.8370383 \\
1 & 2.9274128 & 3.2918405 & 3.9528022 & 2.1870188 & 2.6747397 & 3.4772541 & 1.5785297 & 2.5680523 \\
2 & 3.9414440 & 4.3370543 & 5.0071893 & 3.2260195 & 3.7095838 & 4.4867680 & 2.2966386 & 3.2659358 \\
3 & 4.8433785 & 5.2545579 & 5.9290480 & 4.1395244 & 4.6208542 & 5.3835758 & 2.9834157 & 3.9357442 \\
4 & 5.6693464 & 6.0900511 & 6.7671403 & 4.9722337 & 5.4521361 & 6.2052891 & 3.6435022 & 4.5813401 \\
5 & 6.4394382 & 6.8666546 & 7.5454937 & 5.7467734 & 6.2256717 & 6.9719327 & 4.2804724 & 5.2057558 \\
6 & 7.1660777 & 7.5980685 & 8.2781774 & 6.4765859 & 6.9547326 & 7.6957461 & 4.8971501 & 5.8114252 \\
7 & 7.8575782 & 8.2932428 & 8.9743213 & 7.1704749 & 7.6480344 & 8.3848919 & 5.4958138 & 6.4003383 \\
8 & 8.5198335 & 8.9584266 & 9.6402732 & 7.8345997 & 8.3116853 & 9.0451537 & 6.0783346 & 6.9741474 \\
9 & 9.1572079 & 9.5981991 & 10.2806717 & 8.4734818 & 8.9501754 & 9.6808166 & 6.6462725 & 7.5342431 \\
10 & 9.7730448 & 10.2160418 & 10.8990360 & 9.0905633 & 9.5669262 & 10.2951658 & 7.2009446 & 8.0818094 \\
\hline
\end{tabular}

${ }^{a}$ Exact pseudospin symmetric limit. 


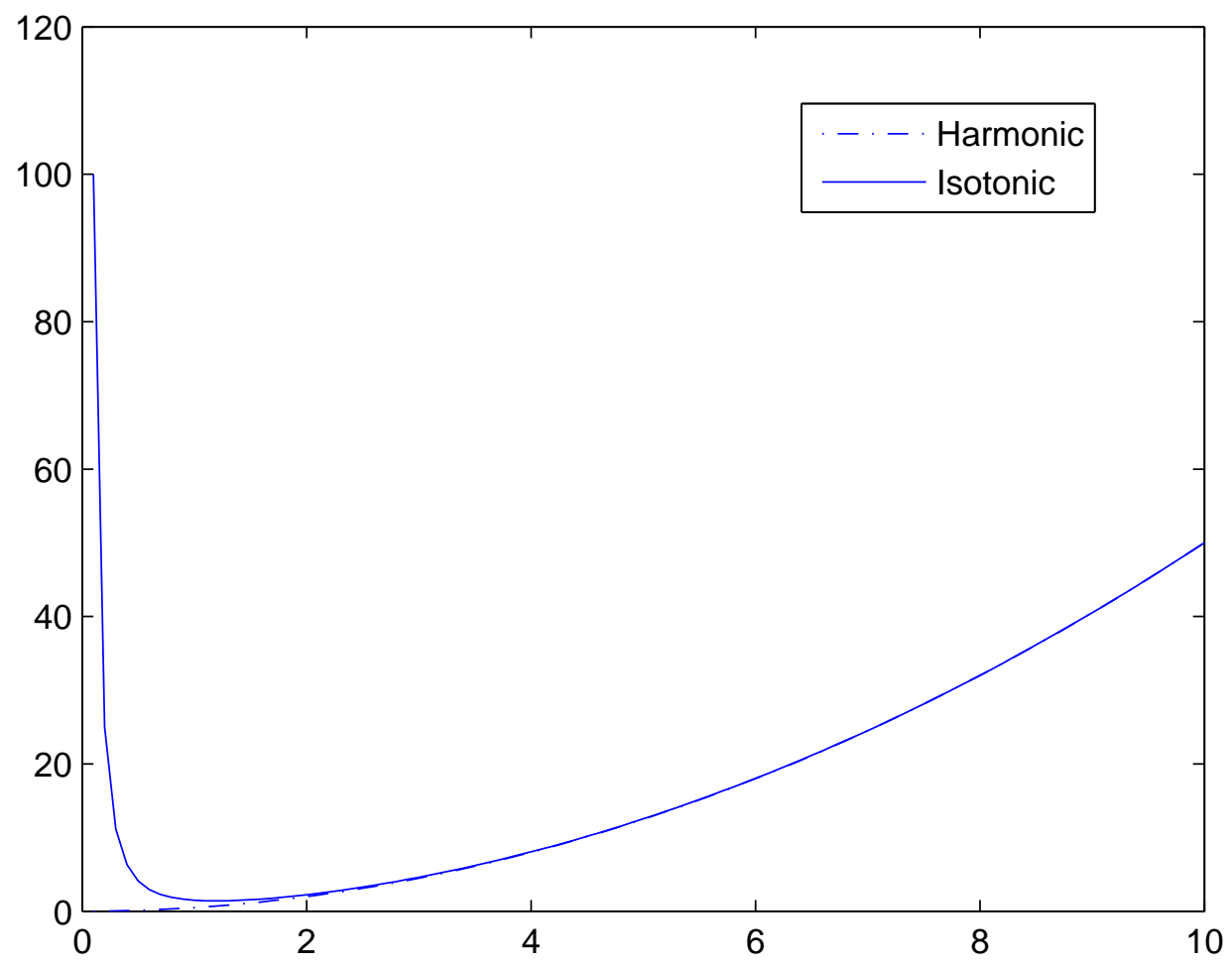




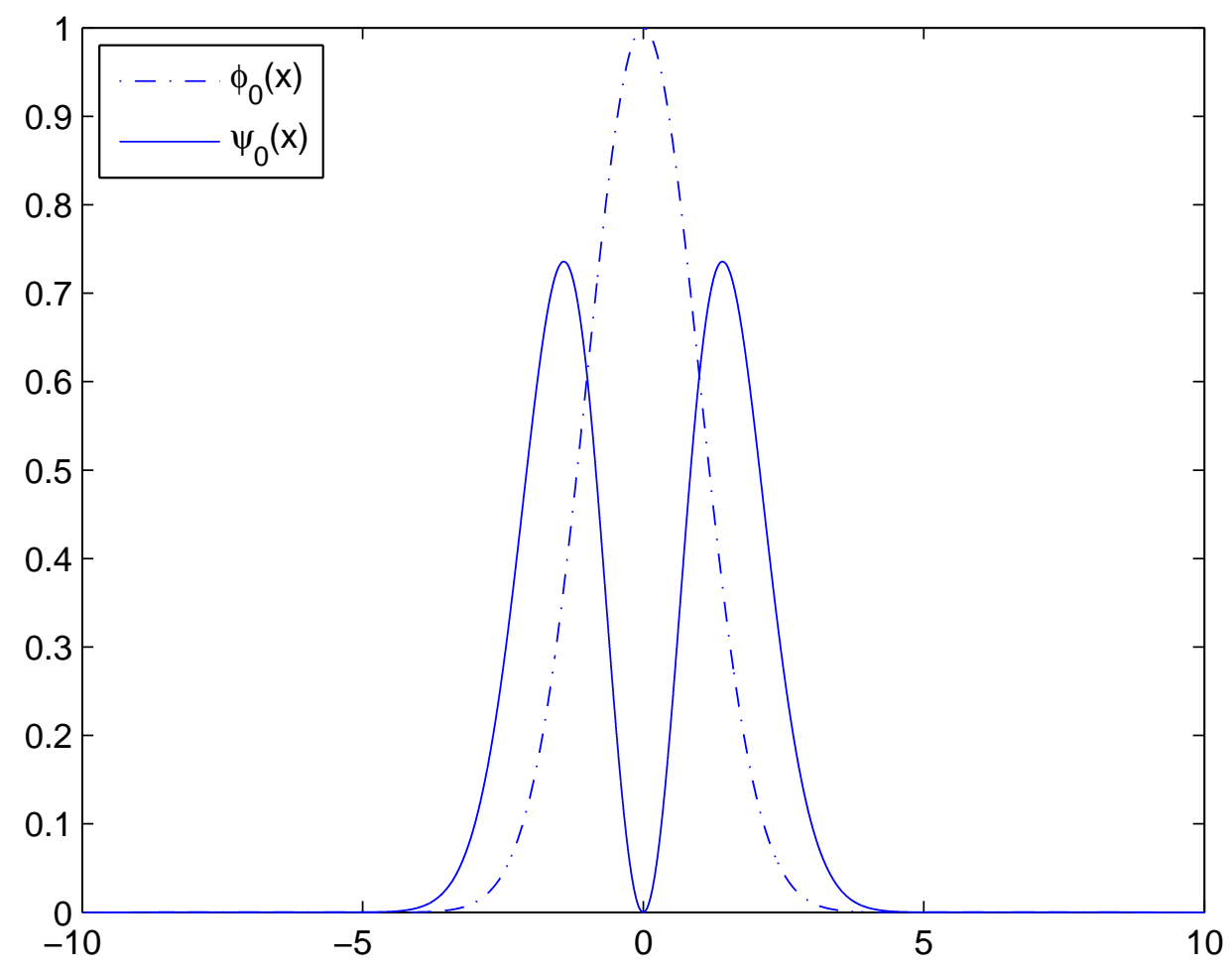




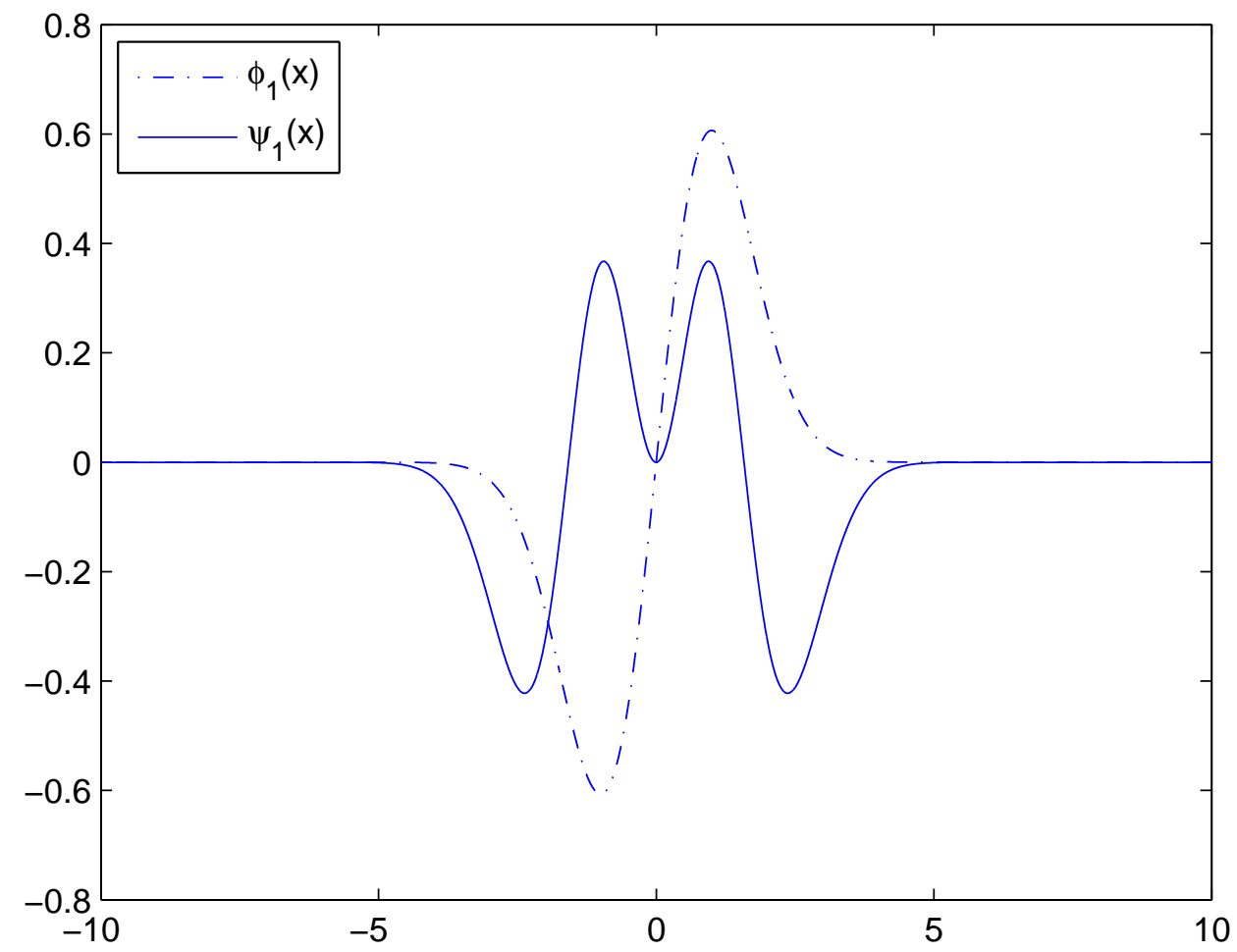




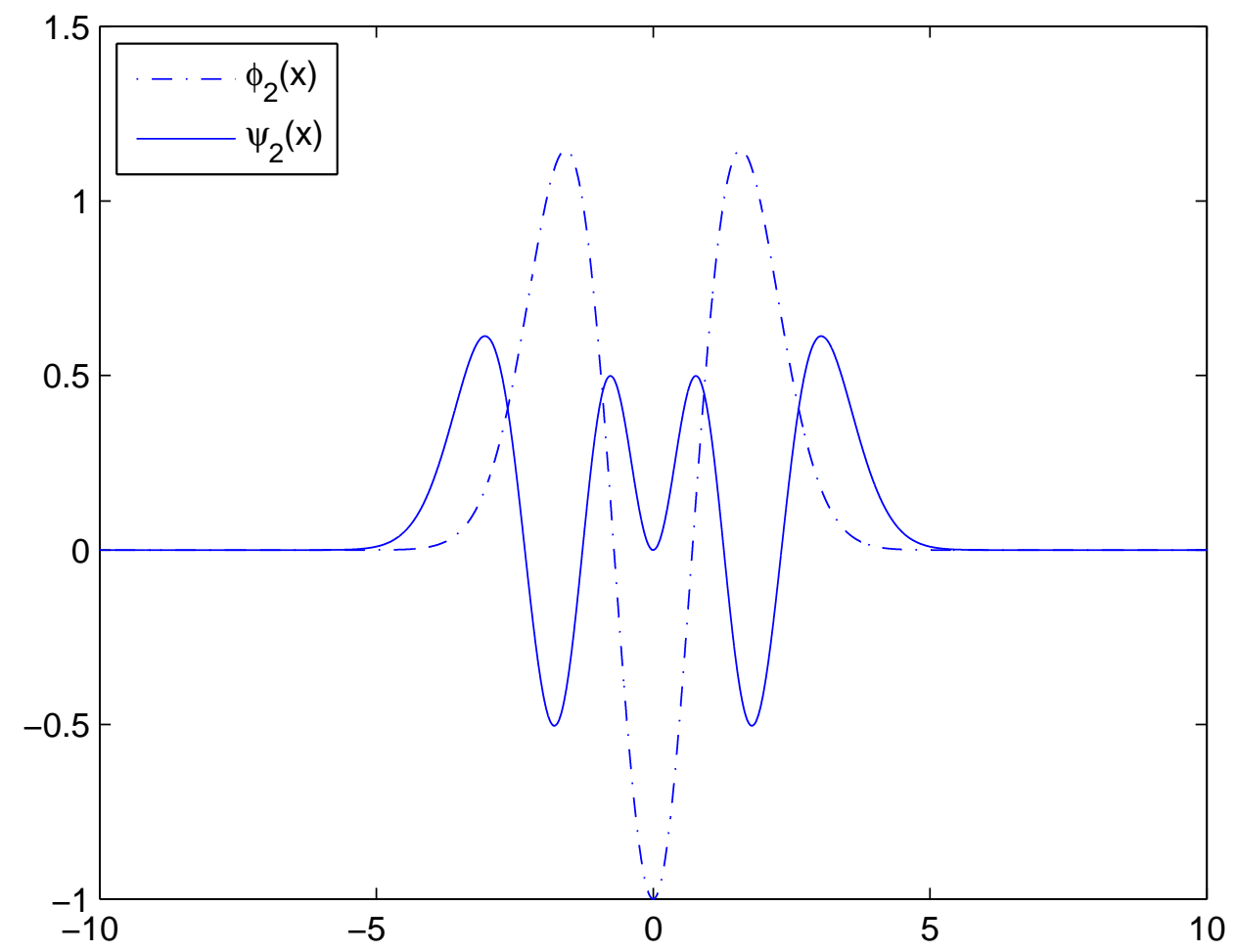

